\title{
A Hybrid ARQ System with Erasures Correction and Parity Retransmission
}

\author{
L. GOLDFELD, A. HOFMAN, V. LYANDRES \\ Department of Electrical and Computer Engineering, Communications Laboratory, \\ Ben-Gurion University of the Negev, Beer-Sheva, Israel \\ Email:lyandres@ee.bgu.ac.il \\ Received June 30, 2009; revised August 4, 2009; accepted September 8, 2009
}

\begin{abstract}
A modified type of Hybrid ARQ system with erasures correction and parity bits retransmission is considered. Performance of the system is analyzed under assumption that the forward channel suffers from Nakagami common fading and additive white Gaussian noise. A good agreement between theoretical results and simulation is achieved. The proposed ARQ protocol is compared with other known Hybrid ARQ algorithms. It demonstrates significantly higher throughput efficiency in a range of SNR.
\end{abstract}

Keywords: Hybrid ARQ Scheme, Erasures Correction Decoding, Fading Channel

\section{Introduction}

Automatic Repeat ReQuest (ARQ) systems with error control are widely used for data transmission over noisy channels. Their performance is usually characterized by two parameters: Throughput Efficiency $(T E)$ and Bit Error Rate $(B E R)$. So-called hybrid ARQ (HARQ) systems use two codes (inner and outer) [1-8]. In a HARQ-I system Forward Error Correction (FEC) is performed prior to error detection [2]. In more effective HARQ-II [3-5] system, parity-check digits for error correction are sent to the receiver only when they are needed. For example [3], at the initial step data blocks $D$ with parity-check bits of the outer error detection code are transmitted. If errors in $D$ are detected, the system begins not simple repetitions of $D$, but repetitions of a parity block $P(D)$ of the inner code. $P(D)$ as well as $D$ itself are alternately stored in the receiver buffer for error correction until $D$ will be recovered. As shown in [4], application of HARQ schemes can significantly improve $T E$ in comparison with a pure ARQ scheme.

It is well known [1] that error correcting capability of block codes may be enhanced when soft decision approach is realized, for example, in the form of Soft Decision Erasures Correcting (SDEC) decoding [7-9]. In this case the number of erroneous bits that may be corrected is not less than $d_{H}-1$. It is assumed also that all error

${ }^{1}$ The assumption of noiseless feedback does not reduce the generality of the analysis, as we are interested in the performance comparison of HARQ-EC and HARQ-II system in the same conditions. symbols are erased, while for FEC decoding the number of erroneous corrected bits is not less than $0.5\left(d_{H}-1\right)$, where $d_{H}$ is the minimum Hamming distance of the block code.

In this paper, a modified type of HARQ-II is considered. Two linear block codes are used in the system: an outer systematic $(n, k)$ block code $C_{2}$ and an inner halfrate invertible $(2 k, k)$ code $C_{1}$. At the receiver FEC and SDEC decoding are used alternately, according to the procedure described below. The system is named HARQ with Erasures Correction (HARQ-EC). Theoretical analysis and computer simulation of the proposed system are performed for the case of noiseless feedback, Nakagami common fading and Additive White Gaussian Noise (AWGN) in the forward channel ${ }^{1}$. Moreover, we consider the forward channel as memoryless, i.e. interleaving/de-interleaving assumed to be ideal. The obtained results show that HARQ-EC provides gain in $B E R$, or gain in $T E$ in comparison with parameters of HARQ-II for the same outer and inner codes [2,4-5].

The paper is organized as follows. In Section 2, we describe the HARQ-EC algorithm. In Section 3 the relevant $B E R$ and $T E$ are analyzed. The comparison of HARQ-EC and HARQ-II characteristics is given in Section 4. Section 5 presents discussion of results and some conclusions.

\section{Description of the HARQ-EC System}

HARQ-EC scheme uses the outer $(n, k)$ code $C_{2}$ with 
minimal Hamming distance $d_{H}^{(2)}$ and the inner $(2 k, k)$ half-rate invertible code [2] $C_{1}$ with minimal Hamming distance $d_{H}^{(1)}$. It is called invertible since with the help of inversion of $k$ parity-check bits $k$ information bits can be uniquely determined. We assume that each transmitted message $D$ consists of $k$ information bits and that each encoded message, called a code word $C W$, consists of $n$ bits. When $D$ is ready for transmission, it is encoded by the outer encoder into the transmitted $n$-length code word $C W_{2}(D, Q)$, where $Q$ denotes the vector of $n-k$ parity-check bits. In parallel the transmitter computes $k$ bits of the parity-check block $P(D)$ of the half rate invertible $(2 k, k)$ code $C_{l}$. The block $P(D)$ is not transmitted and is stored in the buffer for later use.

Let $\mathrm{CW}_{2}(\mathrm{Dr}, \mathrm{Qr})$ denotes the received vector if $C W_{2}(\mathrm{Dr}, \mathrm{Qr})$ was transmitted. The received data block $\mathrm{Dr}$ is passed to the forward channel receiver of the HARQ-EC. Its key elements are Soft Decision Maximum Likelihood Demodulator (SDMLD), FEC and Errors Erasure Correction (EEC) decoders for the outer and inner codes respectively. In SDMLD the decision $A_{l}^{(r)}$ about symbol $\mathrm{Al}$ is obtained according to the following rule [7]:

$$
A_{l}^{(r)}=\left\{\begin{array}{c}
A_{i} \quad \text { if } \max _{k} \Lambda_{k} \\
=\Lambda_{i} \text { and }\left|\Lambda_{i}\right|>\text { thr where } i, k=1,2, . ., M \\
\text { Erasure } \quad \text { if } \max _{k} \Lambda_{k} \\
\quad=\Lambda_{i} \text { and }\left|\Lambda_{i}\right| \leq t h r
\end{array}\right.
$$

where $\Lambda_{i}$ is the log-likelihood ratio calculated for the $i$-th symbol, $M$ is the dimension of the used constellation, and $t h r$ is the threshold level determining the width of the erasure zone in the decision space of the SDMLD. If the number of the erased bits $t_{e r}^{(2)}$ in the code word is zero, the received vector feeds the outer code of the FEC decoder and after error correction the restored message $\mathrm{Dr}$ is sent to the user. If $t_{e r}^{(2)}>0$, the received vector is passed to the outer code of the EEC decoder. If this combination is identified by the EECD with only one of the codebook $C_{2}$, it is considered as the transmitted codeword and the message $\mathrm{Dr}$ is sent to the user. Otherwise, the ReQuest signal (RQ) is sent to the transmitter via the feedback channel. Simultaneously, the message $D r$ (with erased elements) is saved in the buffer of the receiver. Upon receiving this request, the transmitter encodes the $k$-th parity bits block $P(D)$ of the inner code $C_{1}$ into the $n$-length codeword $C W_{2}\left(P(D), Q^{(p)}\right)$ of the code $C_{2}$ where $Q^{(p)}$ denotes the $n-k$ parity-check digits for $P(D)$.

Let $C W_{2}\left(P_{r}(D), Q_{r}^{(p)}\right)$ denotes the received vector corresponding to $C W_{2}\left(P(D), Q^{(p)}\right)$. The SDMLD, according to (1), erases its unreliable symbols. If the number of the erasures $t_{e r}^{(2)}$ in $C W_{2}\left(P_{r}(D), Q_{r}^{(p)}\right)$ is equal to zero, the received vector is passed to the FEC decoder of the outer code. After error correction procedure, the message $D$ is recovered from $P_{r}(D)$. by inversion and is sent to the user. Otherwise, the received vector is passed to the EECD of the outer code. If vector $C W_{2}\left(P_{r}(D), Q_{r}^{(p)}\right)$ is identified by the EECD, the message $D$ is recovered with the help of inversion of $P_{r}(D)$. The message $D r$ that is stored in the receiver memory (after recovery of $D$ from $\left.P_{r}(D)\right)$ is then discarded. If the combination $C W_{2}\left(P_{r}(D), Q_{r}^{(p)}\right)$ is not identified by the EECD of the outer code, the received parity block $P_{r}(D)$ is integrated with $D r$ kept in the buffer. The code word $C W_{1}\left(D_{r}, P_{r}(D)\right)$ of the code $C_{l}$ with $t_{e r}^{(1)}$ erased symbols is formed and passed to EECD of the inner code. If this combination is identified by EECD with certain vector of the code book $C_{1}$ then it is considered to be correct and the recovered message $D$ is passed to the user. Otherwise, the request signal is generated and transmitted via the backward channel. Simultaneously, the message $D$ is discarded from the receiver buffer, and the parity block $P_{r}(D)$ (with erased symbols) is saved in the receiver buffer. Upon receiving the second request for the message $D$, the transmitter resends the code word $C W_{2}(D, Q)$ and the procedure described above is repeated. The block diagram in Figure 1 illustrates transmission and retransmission procedures in the proposed HARQEC.

\section{Analysis of the HARQ-EC Performance in Fading Channel}

The main characteristics of any ARQ systems are BER and TE, defined as

$$
\begin{aligned}
& T E=\frac{E[N]}{E[V]} \cdot \frac{k}{n}, \\
& B E R=1-\left(1-P_{N C}\right)^{k}
\end{aligned}
$$

where $V$ is the total number of transmitted code words and $N$ is the number of information messages sent during the transmission interval, $E[V], E[N]$ denote the expectations of $V$ and $N$ respectively, and $P_{N C}$ is the probability of an undetected word error. As follows from [2], for selective-repeat ARQ scheme with noiseless feedback channel, unlimited receiver buffer and maximal number of retransmissions the values of $T E$ and $P_{N C}$ may be written as

$$
\begin{aligned}
& T E \geq \frac{k}{n}\left(P_{c r d}^{(l)}+P_{\text {erd }}^{(l)}\right) \\
& P_{N C}=\frac{P_{\text {erd }}^{(l)}}{P_{\text {erd }}^{(l)}+P_{\text {crd }}^{(l)}}
\end{aligned}
$$

where $P_{c r d}^{(l)}$ and $P_{\text {erd }}^{(l)}$ are probabilities of correct and erroneous reception of the data block, respectively, at the 


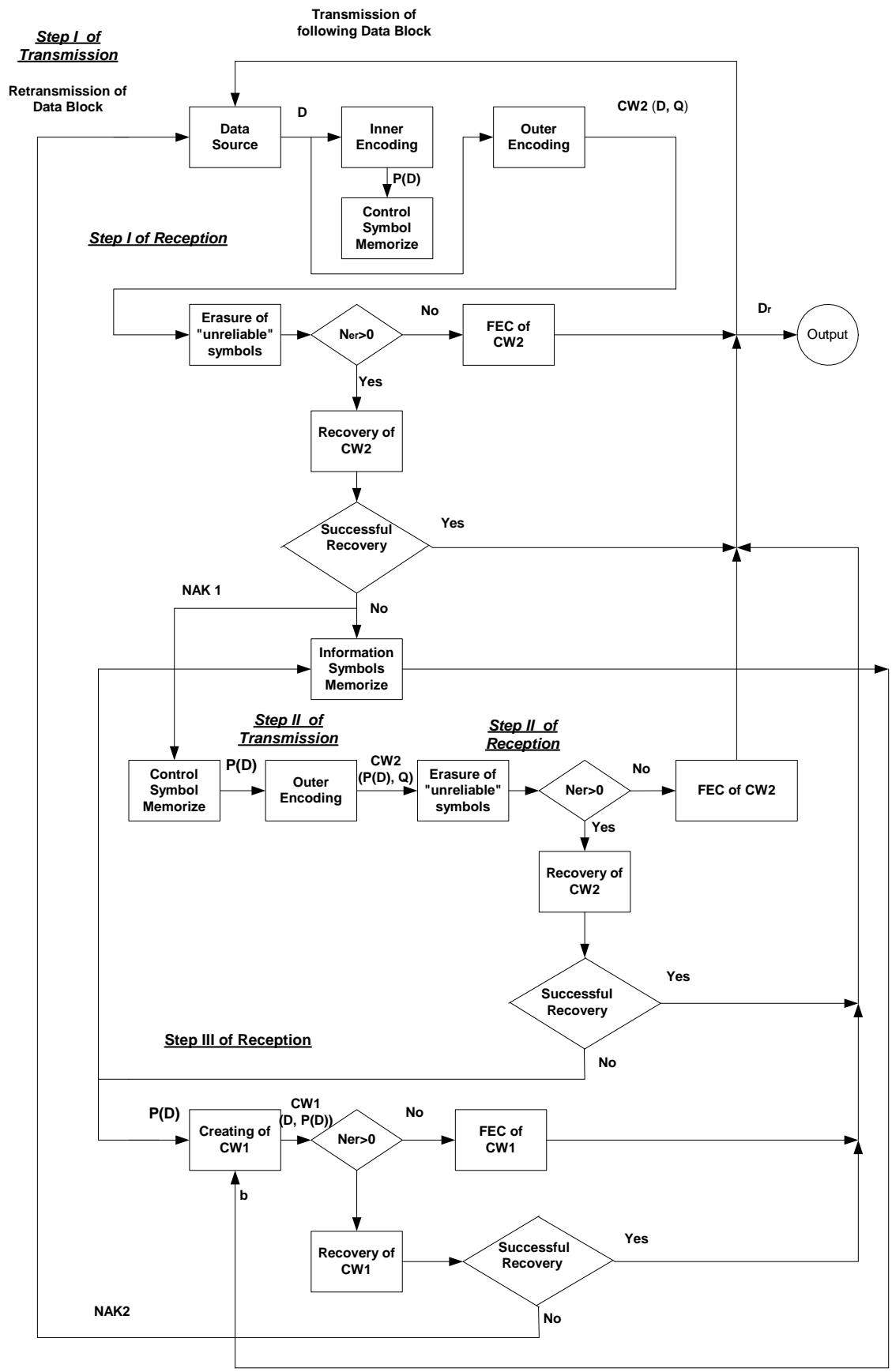

Figure 1. Transmission and retransmission procedures in HARQ-EC.

$l$-th ${ }^{2}$ start of the procedure described above (see Figure 1).

We analyze performance of HARQ-EC for the case of a binary modulation. The transmitted signal within one

${ }^{2}$ The index $l$ will be omitted as the statistics of errors and erases in the received codeword do not depend on $l$.

${ }^{3}$ The kind of modulation and alphabet dimension $M$ does not reduce the generality of the analysis, as we are interested in a comparison of the HARQ-EC performance to HARQ-II systems in the same conditions. symbol time duration $T$ is represented by

$$
s_{m}(t)=\operatorname{Re}\left\{A_{m} g(t-m T) \exp \left(j \omega_{c} t\right)\right\}
$$

where $A_{m}$ is the information symbol, $g(t)$ is the impulse response of the transmitter filter and $w_{c}$ is the carrier frequency ${ }^{3}$. As was mentioned earlier, we consider the case of the forward channel with additive white Gaussian noise and flat fading with Nakagami distribution [8] 


$$
f\left(\mu_{c h}\right)=\frac{2}{\Gamma(m)}\left(\frac{m}{\mu_{0}^{2}}\right)^{m} \mu^{2 m-1} \exp \left(-\frac{m \mu^{2}}{\mu_{0}^{2}}\right)
$$

where $\Gamma(m)$ is the gamma function, $\mu_{0}^{2}=E\left\{\mu^{2}\right\}$, and $\mathrm{m}$ $\geqslant 0.5$ is the fading depth parameter ${ }^{4}$. Moreover, it is assumed that fading is slow, which means that $\mu_{c h}$ may be considered constant, at least for one symbol interval $T$.

The signal $x_{m}(t)$ is demodulated by SDMLD which includes a Log-Likelihood Ratio (LLR) estimator followed by a Decision Device with Eraser (DDE) [1]. The output of LLR is

$$
\Lambda_{12}=q_{1}-q_{2},
$$

where

$$
q_{i}=\int_{0}^{T} x_{m}(t) s_{i}(t) d t, \quad i=1,2
$$

for coherent SDMLD and

$$
q_{i}=\sqrt{X_{i}^{2}+Y_{i}^{2}}, \quad i=1,2
$$

for noncoherent SDMLD.

Here

$$
X_{i}=\int_{0}^{T} x_{m}(t) s_{i}(t) d t, \quad Y_{i}=\int_{0}^{T} x_{m}(t) \hat{s}_{i}(t) d t, \hat{s}_{i}(t) \text { is the }
$$

Hilbert transform of $s_{i}(t)$, and $T$ is the symbol duration. The vector $\boldsymbol{\Lambda}_{\mathbf{c w}}=\left[\boldsymbol{\Lambda}_{1}, \boldsymbol{\Lambda}_{2}, \ldots \boldsymbol{\Lambda}_{l} \ldots, \boldsymbol{\Lambda}_{n}\right]$ is passed to DDE which produces a version of the outer code word. The elements of the received vector $C_{2}^{(r)}$ are obtained according decision rule (1), which in our case can be written as

$$
A_{l}^{(r)}=\left\{\begin{array}{l}
1 \text { if } \Lambda_{l}>t h r \\
0 \text { if } \Lambda_{l}<t h r \\
\text { erase if } 1 / t h r \leq \Lambda_{l} \leq t h r
\end{array}\right.
$$

Using (6) and results of BER analysis for binary orthogonal set of signals [1] probabilities of symbol error $P_{e}$ and symbol erasure $P_{e r s}$ are written as (see Appendix A)

$$
\begin{aligned}
p_{e}= & \frac{m^{m}(1+1 / t h r) \exp [-(t h r-1) / m]}{(1+t h r)\left[m(1+1 / t h r)+\gamma_{0}\right]^{m}} \\
p_{\text {ers }}= & \frac{m^{m}(1+t h r)^{m}}{(1+1 / t h r)\left[m(1+1 / t h r)+\gamma_{0}\right]^{m}} \\
& -\frac{m^{m}(1+1 / t h r) \exp [-(t h r-1) / m]}{(1+t h r)\left[m(1+1 / t h r)+\gamma_{0}\right]^{m}}
\end{aligned}
$$

where $\gamma_{0}=E\left\{\mu^{2} E_{s} / \mu_{0}^{2} N_{0}\right\}$ and $E_{s}$ is the energy of the

\footnotetext{
${ }^{4}$ The Nakagami pdf includes, as a special case, the Rayleigh pdf for
} $m=1$, and can approximate both the Rice and log-normal pdf's [8]. transmitted signal element. With the help of (7) and (8) we estimate performance of the considered system.

Taking into account transmission and retransmission procedures in HARQ-EC, probabilities of correct and erroneous decoding of the code word can be evaluated with the help of the following expressions

$$
\begin{aligned}
& P_{c r d}=P_{c r d}^{(1)}+P_{r q}^{(1)} P_{c r d}^{(2)}+P_{r q}^{(1)} P_{c r d}^{(3)} \\
& P_{\text {erd }}=P_{\text {erd }}^{(1)}+P_{r q}^{(1)} P_{\text {erd }}^{(2)}+P_{r q}^{(1)} P_{\text {erd }}^{(3)}
\end{aligned}
$$

where

$$
\begin{gathered}
P_{c r d}^{(1)}=P_{c r}^{\left(F E C_{2}\right)}\left[C W_{2}\left(D_{r}, Q_{r}\right)\right]+P_{c r}^{\left(R C_{2}\right)}\left[C W_{2}\left(D_{r}, Q_{r}\right)\right], \\
P_{e r d}^{(1)}=P_{e r}^{\left(F E C_{2}\right)}\left[C W_{2}\left(D_{r}, Q_{r}\right)\right]+P_{e r}^{\left(R C_{2}\right)}\left[C W_{2}\left(D_{r}, Q_{r}\right)\right], \\
P_{r q}^{(1)}=P\left(t_{e r}^{(2)} \geq d_{H}^{(2)}\right)
\end{gathered}
$$

are probabilities of correct decoding, erroneous decoding and request of the code word $C W_{2}\left(D_{r}, Q_{r}\right)$ respectively, at the first stage of transmission,

$$
\begin{aligned}
P_{c r d}^{(2)}= & P_{c r}^{\left(F E C_{2}\right)}\left[C W_{2}\left(P_{r}(D), Q_{r}^{(p)}\right)\right] \\
& +P_{c r}^{\left(R C_{2}\right)}\left[C W_{2}\left(P_{r}(D), Q_{r}^{(p)}\right)\right] \\
P_{e r d}^{(2)} & =P_{e r}^{\left(F E C_{2}\right)}\left[C W_{2}\left(P_{r}(D), Q_{r}^{(p)}\right)\right] \\
& +P_{e r}^{\left(R C_{2}\right)}\left[C W_{2}\left(P_{r}(D), Q_{r}^{(p)}\right)\right]
\end{aligned}
$$

are probabilities of correct decoding and erroneous decoding of the codeword $C W_{2}\left(D_{r}, Q_{r}\right)$ respectively, at the second stage of transmission,

$$
\begin{aligned}
P_{c r d}^{(3)}= & P_{c r}^{\left(F E C_{1}\right)}\left[C W_{1}\left(D_{r}, P_{r}(D)\right)\right] \\
& +P_{c r}^{\left(R C_{1}\right)}\left[C W_{1}\left(D_{r}, P_{r}(D)\right)\right] \\
P_{\text {erd }}^{(3)} & =P_{e r}^{\left(F E C_{1}\right)}\left[C W_{1}\left(D_{r}, P_{r}(D)\right)\right] \\
& +P_{e r}^{\left(R C_{1}\right)}\left[C W_{1}\left(D_{r}, P_{r}(D)\right)\right]
\end{aligned}
$$

are probabilities of correct and erroneous decoding, respectively, of the code word $C W_{1}\left(D_{r}, P_{r}(D)\right)$, which is created from the block of data $D r$ extracted from the receiver memory and the parity block $P_{r}(D)$ of the inner code received at the second stage of transmission. Here $P_{c r}^{\left(F E C_{j}\right)}, P_{e r}^{\left(F E C_{j}\right)}$ are probabilities of correct and erroneous reception of code words at the output of the FEC decoders, and $P_{c r}^{\left(R C_{j}\right)}, P_{e r}^{\left(R C_{j}\right)}$ are probabilities of correct and erroneous reception of code words at the output of the errors erasure correction decoders, for the inner $(j=1)$ and outer $(j=2)$ codes respectively.

Bearing in mind the assumptions made above on the statistical properties of the channel, we consider that errors, as well as erasures in the received stream of code 
symbols, are independent. In this case probabilities $P_{c r}^{\left(F E C_{j}\right)}, P_{e r}^{\left(F E C_{j}\right)}, P_{c r}^{\left(R C_{j}\right)}, P_{e r}^{\left(R C_{j}\right)}$ and $P_{r q}^{(j)}$ for the outer and inner codes can be determined (Appendix B) with the help of the following expressions:

$$
\begin{aligned}
& { }_{c r}^{\left(F E C_{j}\right)}=\left(1-p_{e r s}\right)^{n_{j}} \sum_{i=0}^{t_{c_{j}}}\left(\begin{array}{l}
n_{j} \\
i
\end{array}\right) p_{e}^{i}\left(1-p_{e}\right)^{n_{j}-i}, \\
& P_{e r}^{\left(F E C_{j}\right)}=\left(1-p_{e r s}\right)^{n_{j}} \sum_{i=t_{c_{j}}}^{n_{j}}\left(\begin{array}{c}
n_{j} \\
i
\end{array}\right) p_{e}^{i}\left(1-p_{e}\right)^{n_{j}-i}, \\
& P_{r q}^{(j)}=\sum_{i=d_{H_{j}}}^{n_{j}}\left(\begin{array}{c}
n_{j} \\
i
\end{array}\right) p_{\text {ers }}^{i}\left(1-p_{\text {ers }}\right)^{n_{j}-i}, \\
& P_{c r}^{\left(R C_{j}\right)}=\sum_{t_{e r_{j}}=1}^{d_{H_{j}}-1}\left(\begin{array}{c}
n_{j} \\
t_{e r j}
\end{array}\right) p_{e r s}^{t_{e r j}}\left(1-p_{e r s}\right)^{n_{j}-t_{e r j}} \\
& \left\{\sum_{t_{\text {erd }}=0}^{t_{\text {er }}}\left(\begin{array}{c}
t_{e r_{j}} \\
t_{\text {erd }}
\end{array}\right) p_{\text {ers }}^{t_{\text {erd }}}\left(1-p_{\text {ers }}\right)^{t_{e_{j} r_{j}}-t_{\text {erd }}} P\left(c r / t_{\text {er }}, t_{\text {erd }}\right)\right\} \\
& P_{e r}^{\left(R C_{j}\right)}=\sum_{t_{e r_{j}}=1}^{d_{H_{j}}-1}\left(\begin{array}{c}
n_{j} \\
t_{e j}
\end{array}\right) p_{e r s}^{t_{e r j}}\left(1-p_{e r s}\right)^{n_{j}-t_{e r j}} \\
& \left\{\sum_{t_{\text {erd } j}=0}^{t_{\text {er }}}\left(\begin{array}{c}
t_{e^{\prime} r_{j}} \\
t_{\text {erd }}
\end{array}\right) p_{\text {erd }}^{t_{\text {erd }}}\left(1-p_{\text {ers }}\right)^{t_{\text {erj }}-t_{\text {erd }}} P\left(e r / t_{e_{j}}, t_{\text {erd }}\right)\right\}
\end{aligned}
$$

where

$$
\begin{aligned}
& P\left(\operatorname{cr} / t_{e_{j}}, t_{e r d_{j}}\right)=\sum_{i=0}^{0.5\left(d_{H_{j}}-t_{e r d j}-1\right)}\left(\begin{array}{c}
n_{j}-t_{e r j} \\
i
\end{array}\right) p_{e}^{i}\left(1-p_{e}\right)^{n_{j}-t_{e r_{j}}-i} \\
& P\left(e r / t_{e_{j}}, t_{e r d_{j}}\right)=\sum_{i=0.5\left(d_{H_{j}}-t_{e r d_{j}}+1\right)}^{n_{j}-t_{e r j}}\left(\begin{array}{c}
n_{j}-t_{e r j} \\
i
\end{array}\right) p_{e}^{i}\left(1-p_{e}\right)^{n_{j}-t_{e r j}-i}
\end{aligned}
$$

$n_{j}$ is the length of code word, $d_{H_{j}}$ is the minimum Hamming distance of the used code, $t_{e r_{j}}$ is a number of codeword symbols erased in the SDMLD, and $t_{\text {erd }}$ is the number of the erased symbols taken from those that determine the Hamming distance of the original code $\left(0 \leq t_{e^{2} d_{j}} \leq t_{e r_{j}}\right)$. Substituting (7), (8) into (13), (14), and the results into (9-12) and afterward into (2) and (3), we obtain values of $T E$ and $B E R$. For the given inner and outer codes they depend on the average SNR $\gamma_{0}$ and on the threshold thr.

\section{Comparison of HARQ-EC and HARQ-II Performance}

In this Section, we compare the theoretical results obtained above for HARQ-EC with those obtained by

\footnotetext{
${ }^{5}$ The kind of modulation and type of codes do not reduce the generality of the analysis, as we are interested in comparison of the HARQ-EC performance to HARQ-II systems in the same conditions.
}

computer simulation. Then we will compare the performance of HARQ-EC to that of HARQ-II schemes [2, $4,5]$ for the same inner and outer codes ${ }^{5}$. The systematic linear block code with parameters $n_{2}=15, k=11$ and $d_{H_{2}}=3$ is used as the outer code $C_{2}$, and the half invertible code with $n_{1}=22, k=11$ and $d_{H_{1}}=7$ is used as the inner code $C_{1}$.

Figures 2, 3 show for HARQ-EC dependence of $B E R$ and $T E$ (for the threshold values $t h r=1.5$, thr $=2$, and $t h r=3$ ), obtained as a result of analytical calculation and computer simulation respectively. Inspection of Figures 2 and 3 demonstrates good agreement between theory and simulation results. It follows that increase of the threshold level thr in HARQ-EC leads to decrease of both $B E R$ and $T E$.

Figure 4 shows $B E R$ for HARQ-EC (for the threshold values $t h r=1.5$, $t h r=2$, and $t h r=3$ ), HARQ-II and FEC systems with the same code redundancy as a function of the average SNR while at Figure 5 dependence of $T E$ of the compared systems from average SNR is presented.

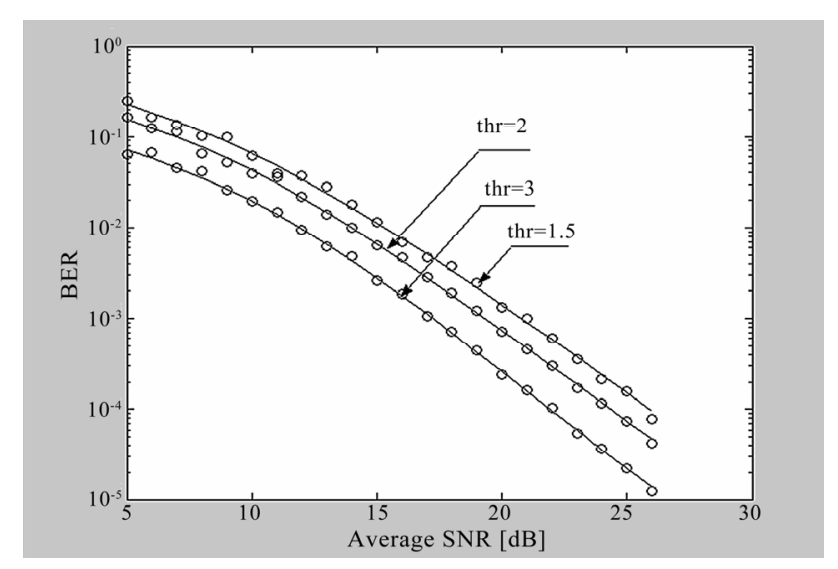

Figure 2. Average BER of HARQ-EC2 as a function of the average SNR (for the threshold values $t h r=1.5$, thr=2, and $t h r=3)$; analytical calculation and computer simulation.

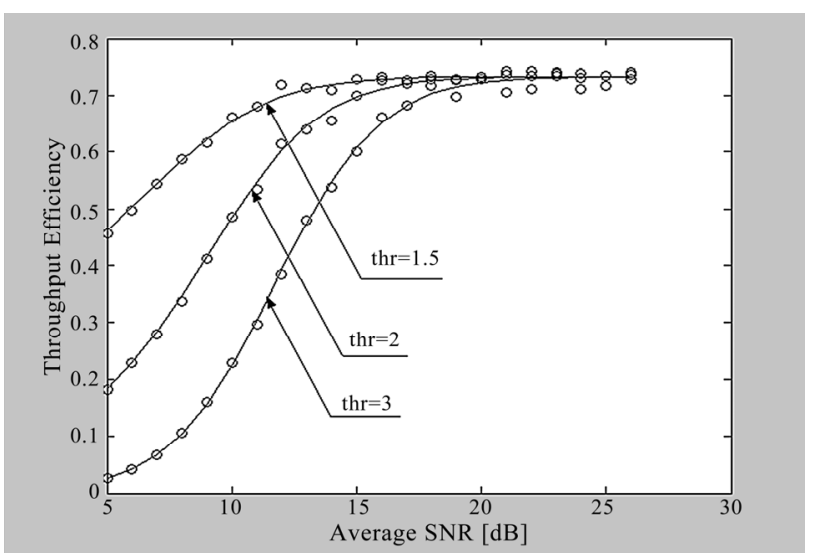

Figure 3. Throughput efficiency of HARQ-EC2 as a function of average $\mathrm{SNR}$ (for the threshold values $t h r=1.5$, thr $=2$, and $t h r=3$ ); analytical calculation and computer simulation. 


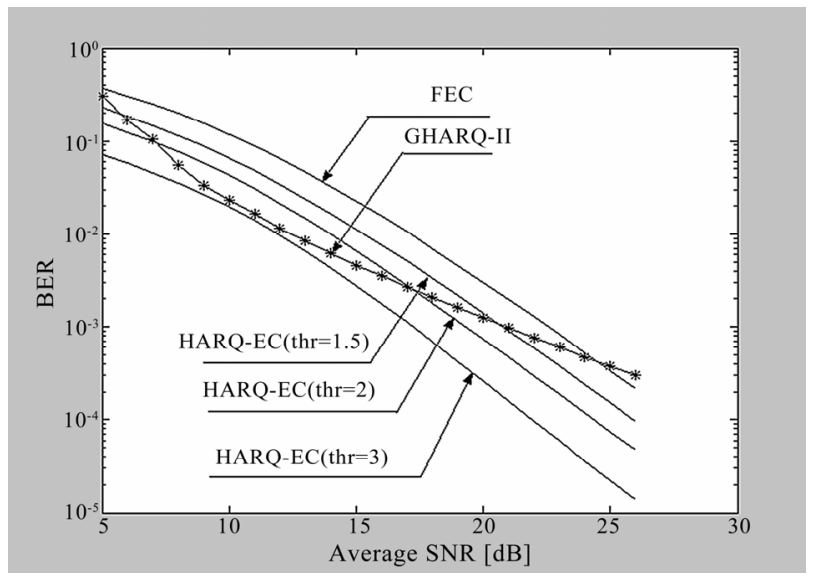

Figure 4. Average BER of HARQ-EC and HARQ-II systems as functions of average SNR.

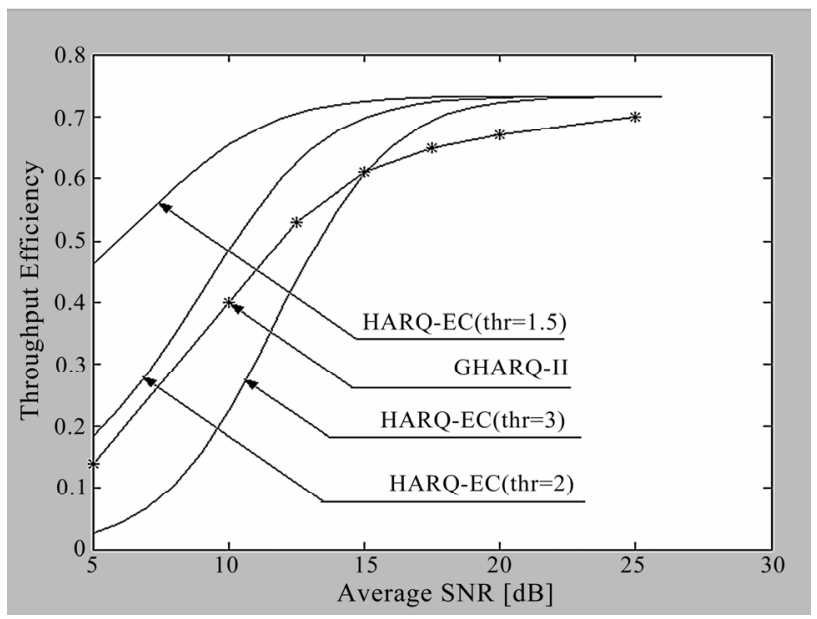

Figure 5. Throughput efficiency of HARQ-EC and HARQII systems as functions of average SNR.

Examination of these figures demonstrates the fact that by choice of $t h r$ value in SDMLD of HARQ-EC, gain in $B E R$ or $T E$ can be achieved. For example, $T E$ of HARQ-EC with $t h r=2$ exceeds $T E$ of HARQ-II for a roughly equal value of $B E R$ in a quite wide range of average SNR.

\section{Conclusions}

We propose the Hybrid ARQ system with erasure of un- reliable symbols and retransmission of the code words (HARQ-EC). Its performance is considered for the case of flat Nakagami fading and AWGN in the forward channel. The obtained theoretical results are valid for any memoryless channel with common slow Nakagami fading, while the calculations and simulation were performed for Rayleigh fading. Good agreement between theoretical and simulation results is obtained. It has been shown that performance of HARQ-EC may be better than HARQ-II over a wide range of average SNR when the same codes are used.

\section{References}

[1] J. C. Proakis, "Digital communications," McGraw-Hill, New York, 1995.

[2] S. Lin and P. S. Yu, "A hybrid ARQ scheme with parity retransmission for error-control in satellite channels," IEEE Trans. Commun., Vol. COM-30, No. 7, pp. 17011719, 1982.

[3] Y. Wang and S. Lin, “A modified Selective-Repeat Type-II Hybrid ARQ system and its performance analysis," IEEE Trans. Commun., Vol. COM-31, No. 5, pp. 593-607, 1983.

[4] K. Q. Archer and J. A. Edwards, "Effect of channel fade rate on throughput of three GHARQ-II schemes over Rayleigh fading channel," Electronic Letters, Vol. 31, No. 16, pp. 1320-1322, 1995.

[5] C. Sunkyun and K. Shin, "A class of adaptive hybrid ARQ for wireless links," IEEE Trans. Veh. Tech., Vol. 50, No. 3, pp. 777-790, 2001.

[6] S. Kallel, R. Link, and S. Bakhtiyari, "Throughput performance of memory ARQ schemes," IEEE Trans. Veh. Tech., Vol. 48, No. 3, pp. 891-899, 1999.

[7] S. B. Wicker, "Reed-Solomon error coding for Rayleigh fading channels with feedback," IEEE Trans. Veh. Tech., Vol. 41, No. 2, pp. 124-133, 1992.

[8] L. Goldfeld, V. Lyandres, and D. Wulich, "ARQ with erasures correction in the frequency-nonselective fading channel," IEICE Trans. Commun., Vol. E80-B, No. 7, pp. 1101-1103, 1997.

[9] T. Hashimoto, "Comparison of erasure-and error threshold decoding schemes," IEICE Trans. Fundamentals, Vol. E76-A, pp. 820-827, 1993. 


\section{Appendix A}

Let us assume that symbol "1" was transmitted. The probability of correct reception $P_{c r}$ in SDMLD can be found as the probability that $\Lambda_{i}>t h r$, and the probability of symbol erasure $P_{\text {ers }}$ in SDMLD, in turn, can be obtained as the probability that $\frac{1}{t h r} \leq \Lambda_{i} \leq t h r$. From (6) we obtain.

$$
\begin{aligned}
& p_{c r}(\mu)=p\left(\Lambda_{i}>t h r\right), \\
& p_{\text {ers }}(\mu)=p\left(\frac{1}{t h r} \leq \Lambda_{i} \leq t h r\right)
\end{aligned}
$$

where (see [1])

$$
\begin{aligned}
& \Lambda_{i}=\frac{U_{1}}{U_{2}} ; \\
& U_{1}=\left|2 E_{S} \mu e^{-j \phi}+N_{1}\right| \\
& U_{2}=\left|N_{2}\right|
\end{aligned}
$$

$N_{1}$ and $N_{2}$ are complex-valued Gaussian random variables with zero mean and variance $\sigma^{2}=2 \mu E s, U_{1}$ and $U_{2}$ are mutually independent variables with distributions [1]

$$
\begin{gathered}
p\left(U_{1}\right)=\frac{U_{1}}{2 E_{S} N_{0}} \exp \left(-\frac{U_{1}^{2}+4 \mu^{2} E_{S}^{2}}{4 E_{S} N_{0}}\right) I_{0}\left(\frac{\mu U_{1}}{N_{0}}\right), \\
\mathrm{p}\left(\mathrm{U}_{2}\right)=\frac{U_{2}}{2 E_{S} N_{0}} \exp \left(-\frac{U_{2}^{2}}{4 E_{S} N_{0}}\right) .
\end{gathered}
$$

Taking into account (A1), (A2) we obtain

$$
\begin{aligned}
& p_{c r}(\mu)=p\left(U_{1}>t h r \cdot U_{2}\right) \\
&= \int_{0}^{\infty} p\left(U_{1}\right) \cdot\left[\int_{0}^{U_{1} / t h r} p\left(U_{2}\right) d U_{2}\right] d U_{1} ; \\
& p_{\text {ers }}(\mu)=p\left(\frac{U_{2}}{\text { thr }} \leq U_{1} \leq t h r \cdot U_{2}\right) \\
&=\int_{0}^{\infty} p\left(U_{1}\right)\left[\int_{U_{1} / t h r}^{U_{1} \text { th } r} p\left(U_{2}\right) d U_{2}\right] d U_{1}
\end{aligned}
$$

With the help of elementary algebra and tabulated integrals

$$
\begin{aligned}
p_{c r}(\mu)= & 1-\frac{t h r}{1+t h r} \exp \left(-\frac{1-t h r}{\gamma(\mu) t h r}\right) \exp \left(-\frac{\gamma(\mu)}{1+t h r}\right) \\
p_{\text {ers }}(\mu)= & \frac{t h r}{1+t h r} \exp \left(-\frac{1-t h r}{\gamma(\mu) t h r}\right) \cdot \exp \left(-\frac{\gamma(\mu)}{1+t h r}\right)- \\
& -\frac{1}{1+t h r} \exp \left(-\frac{t h r-1}{\gamma(\mu)}\right) \cdot \exp \left(-\frac{\gamma(\mu) t h r}{1+t h r}\right)
\end{aligned}
$$

where

$$
\gamma(\mu)=\frac{\mu^{2} E_{S}}{N_{0}}
$$

Taking into account that

$$
p_{e}=1-p_{c r}-p_{e r s}
$$

we have

$$
p_{e}(\mu)=\frac{1}{1+t h r} \exp \left(-\frac{t h r-1}{\gamma(\mu)}\right) \exp \left(-\frac{\gamma(\mu) t h r}{1+t h r}\right)
$$

Probabilities (A6) and (A8) are conditional probabilities of erasure and error reception in SDMLD respectively, given $\mu$ and therefore $\mathrm{Pe}$ and $\mathrm{Per}$ are

$$
\begin{aligned}
& P_{e r}=\int_{0}^{\infty} p_{\text {ers }}(\mu) f(\mu) d \mu, \\
& P_{e}=\int_{0}^{\infty} p_{e}(\mu) f(\mu) d \mu,
\end{aligned}
$$

where $f(\mu)$ is defined by $(5)$.

\section{Appendix B}

Let us determine probability of request $P_{r q}$, probabilities of correct and erroneous reception of a code word at the output of the ECE decoder $\left(P_{c r}^{(R C)}\right.$ and $\left.P_{e r}^{(R C)}\right)$, and also at the output of the FEC decoder $\left(P_{c r}^{(F E C)}\right.$ and $\left.P_{e r}^{(F E C)}\right)$ under the following conditions:

1) The code used is a linear block code $(n, k)$ with the minimal Hamming distance $d_{H}$;

2) The encoded bit stream is represented by a codeword $C W$ with length $n$, supplied from the SDMLD output to the FEC decoder, if $C W$ does not contain the erased symbols. Otherwise, a codeword $C W$ feeds the ECE decoder.

3) Errors and erasers in a sequence of code symbols $\mathrm{CW}$ are independent (the channel is memoryless). For Nakagami frequency -nonselective fading in the forward channel, the probabilities of symbol error $P e$ and symbol erasure Pers are defined by (7), (8).

First, we find probabilities $P_{c r}^{(F E C)}$ and $P_{e r}^{(F E C)}$. Since symbol errors are independent events, the binomial law determines probabilities of correct and erroneous reception of a code word at the output of the FEC decoder. Keeping in mind that FEC decoding is used when the number of erased symbols in the received codeword $t_{e r}=0$, we thus obtain

$$
\begin{aligned}
& P_{c r}^{(F E C)}=\left(1-p_{e r s}\right)^{n} \sum_{i=0}^{t_{c r}}\left(\begin{array}{l}
n \\
i
\end{array}\right) p_{e}^{i}\left(1-p_{e}\right)^{n-i} \\
& P_{e r}^{(F E C)}=\left(1-p_{e r s}\right)^{n} \sum_{i=t_{c r}+1}^{n}\left(\begin{array}{l}
n \\
i
\end{array}\right) p_{e}^{i}\left(1-p_{e}\right)^{n-i}
\end{aligned}
$$


where $t_{c r}$ is the number of correct errors per codeword.

Probabilities $P_{r q}, P_{c r}^{(R C)}$ and $P_{e r}^{(R C)}$ may be obtained as follows. The ECE decoding is used when $t_{e r}>0$, where $t_{e r}$ is a number of the erased symbols in the received code word. The erasure of $t_{e r}$ symbols from $n$ creates a new shorter code with code word length $n^{(s h)}=n-t_{e r}$ and the Hamming distance $d_{H}^{(s h)}=d_{H}-t_{\text {erd }}$, where $t_{\text {erd }}$ is the number of erased symbols taken from those ones that determine $d_{H}$ of the original code $\left(0 \leqslant t_{e r d} \leqslant t_{e r}\right)$. Since symbol erasures are independent, the probability of the erasure of $t_{e r}$ symbols from $n$ as well as probability of the erasure of $t_{\text {erd }}$ symbols from $d_{H}$ are determined by the binomial law. Taking this into account, we obtain $P_{r q}$ as

$$
P_{r q}=P\left(t_{e r} \geq d_{H}\right)=\sum_{t_{e r}=d_{H}}^{n}\left(\begin{array}{c}
n \\
t_{e r}
\end{array}\right) p_{e r s}^{t_{e r}}\left(1-p_{e r s}\right)^{n-t_{e r}}
$$

Probabilities of correct and erroneous reception of a code word at the output of ECE decoder depend on the random variables $t_{e r}$ and $t_{e r d}$, i.e. they have to be considered as conditional probabilities $P\left(c r / t_{e r}, t_{e r d}\right)$ and $P\left(e r / t_{e r}, t_{e r d}\right)$ written as

$$
\begin{aligned}
& P\left(c r / t_{e r}, t_{\text {erd }}\right)=\sum_{i=0}^{0.5\left(d-t_{\text {erd }}-1\right)}\left(\begin{array}{c}
n-t_{e r} \\
i
\end{array}\right) p_{e}^{i}\left(1-p_{e}\right)^{n-t_{e r}-i} \\
& P\left(e r / t_{e r}, t_{e r d}\right)=\sum_{i=0.5\left(d-t_{e r d}+1\right)}^{n-t_{e r}}\left(\begin{array}{c}
n-t_{e r} \\
i
\end{array}\right) p_{e}^{i}\left(1-p_{e}\right)^{n-t_{e r}-i}
\end{aligned}
$$

Twice averaging (B3) by the binomially distributed $t_{e r}$ and $t_{\text {erd }}$, we obtain the unconditional probabilities $P_{c r}^{(R C)}$ and $P_{e r}^{(R C)}$ (see 13). 